\title{
The role of spatial working memory in visual search efficiency
}

\author{
SEI-HWAN OH and MIN-SHIK KIM \\ Yonsei University, Seoul, Korea
}

\begin{abstract}
Many theories have proposed that visual working memory plays an important role in visual search. In contrast, by showing that a nonspatial working memory load did not interfere with search efficiency, Woodman, Vogel, and Luck (2001) recently proposed that the role of working memory in visual search is insignificant. However, the visual search process may interfere with spatial working memory. In the present study, a visual search task was performed concurrently with either a spatial working memory task (Experiment 1) or a nonspatial working memory task (Experiment 2). We found that the visual search process interfered with a spatial working memory load, but not with a nonspatial working memory load. These results suggest that there is a distinction between spatial and nonspatial working memory in terms of interactions with visual search tasks. These results imply that the visual search process and spatial working memory storage require the same limited-capacity mechanisms.
\end{abstract}

Efficiently finding an item of relevance among the vast amounts of information around us is crucial, especially if one considers the limited capacity of the human visual system. Many theories have proposed that efficient search is possible because of the close interaction between attention and working memory. Supporting evidence for this can be found in the studies with visual search tasks reviewed below.

Visual search paradigms have been used extensively to investigate attentional selection in the visual system. In a typical visual search task, participants report whether a given scene contains a predefined target stimulus among a set of distractors. When the target is difficult to find, search response times (RTs) increase linearly with increases in search set size, and search slopes are approximately twice as steep when the target is absent, as compared with when it is present. On the basis of the assumption that items are usually not reinspected during a trial, this phenomenon has been interpreted to be the result of a serial self-terminating search process involving focal attention (Cave \& Wolfe, 1990; Treisman \& Gelade, 1980; Wolfe, 1994). This assumption has been supported by numerous studies providing evidence that information about items searched accumulates in the memory system (for a review, see Shore \& Klein, 2000).

A portion of the present study was presented at the second annual meeting of the Asian Conference on Vision, Gyeongju, Korea, 2002. The present study was supported by Korea Research Foundation Grant KRF-2002-074-HS 1003. We thank Chan-Sup Chung, Keetaek Kham, Marvin Chun, and Helena Paterson for continuous interest in and helpful comments on the present study. We thank Paul E. Downing, Cathleen M. Moore, Geoffrey F. Woodman, and an anonymous reviewer for their valuable comments. Correspondence concerning this article should be addressed to M.-S. Kim, Department of Psychology, Yonsei University, Seoul 120-749, Korea (e-mail: kimm@yonsei.ac.kr).
Moreover, Desimone and Duncan (Desimone, 1996, 1998; Desimone \& Duncan, 1995) have proposed that working memory influences the allocation of selective attention. This proposal has been strongly supported by cell recordings in the inferior temporal cortex of macaque monkeys during visual search task, in which neuronal activities were biased in favor of the stimulus held in working memory (Chelazzi, Duncan, Miller, \& Desimone, 1998; Chelazzi, Miller, Duncan, \& Desimone, 1993). Consistent with this, recent work with human observers has shown that an object in working memory shifts selective attention toward that object even though there is little reason to select it (Downing, 2000). In this study, a memory stimulus and a novel stimulus were followed by a probe discrimination task. The results showed that processing of the probe was faster when it was presented at the location of the memory stimulus than when it was presented at the location of a novel stimulus, indicating that the stimulus held in working memory received more attention.

Together, the findings above indicate that the visual search process is strongly influenced not only by selective attention, but also by working memory. Therefore, when a visual search task is performed with an additional visual working memory task, interference between the two tasks is expected to occur. However, Woodman, Vogel, and Luck (2001) have recently reported results that are inconsistent with this prediction. In their study, participants performed a visual search task while maintaining four colors or shapes in visual working memory. Maintaining the four objects in visual working memory was regarded as filling visual working memory to its capacity (Luck \& Vogel, 1997). Therefore, if the visual search process were to interfere with working memory, the accuracy of the working memory task should decrease as search set size increases, and the memory load should 
impair search efficiency. Since a task switch increases search RTs constantly across all search set sizes, an impairment in search efficiency should be measured as an increase in search rate (i.e., the slope created by RTs as a function of search set size), rather than as an increase in overall search RT. However, working memory load did not impair search efficiency, and the visual search process did not impair memory accuracy.

The purpose of the present study was to reinvestigate the interactions between visual selective attention and visual working memory, using Woodman et al.'s (2001) dual-task paradigm. Whereas Woodman et al. used only nonspatial working memory tasks to examine the role of visual working memory in visual search, we compared the interaction of selective attention with spatial working memory (Experiment 1) and with nonspatial working memory (Experiment 2) during visual search. There is a dissociation between spatial and nonspatial working memory (Baddeley \& Logie, 1999; Courtney, Petit, Maisog, \& Ungerleider, 1998; Courtney, Ungerleider, Keil, \& Haxby, 1996; McCarthy, et al., 1996; Smith, et al., 1995), and spatial processing has been implicated during visual search tasks (Kim \& Cave, 1995, 1999; Kim \& Robertson, 2001; Treisman \& Gelade, 1980). Thus, interactions between visual search and visual working memory could vary depending on which type of visual working memory is examined.

In addition, we made the search task more difficult than that in Woodman et al. (2001), in order to ensure that focal attentive scrutiny of each stimulus would be required. To measure the degree of memory impairment more precisely, we also changed the method for testing the performance of working memory. Whereas Woodman et al. used a recognition memory task in which participants indicated whether the memory test array was identical to the memory array, we presented only one memory test probe. For the present working memory task, the participants indicated whether the location (Experiment 1) or the color (Experiment 2) of the memory test probe was the same as that for one of the four memory stimuli (Smith, Jonides, \& Koeppe, 1996).

\section{EXPERIMENT 1 Spatial Working Memory and Visual Search}

\section{Method}

\section{Participants}

Seventeen undergraduate students with normal or corrected-tonormal vision participated for course credit after giving informed consent.

\section{Stimuli and Tasks}

The stimuli were presented in a $7.82^{\circ} \times 7.82^{\circ}$ of visual angle display field $\left(9.9 \mathrm{~cd} / \mathrm{m}^{2}\right)$ at a viewing distance of $57 \mathrm{~cm}$ within an imaginary square grid of 64 cells. A fixation cross was presented continuously at the center of the display field (see Figure 1).

Working memory task. The memory stimuli for each trial were four black filled squares (memory stimuli, each $0.4^{\circ} \times 0.4^{\circ}$ of visual angle). The location of the filled squares was selected randomly from eight possible locations, which were $0.68^{\circ}$ of visual angle from fixation. The participants were asked to remember the locations of the four squares in the memory array until the end of the trial.

At the end of each trial, one black empty square (memory test probe, $0.46^{\circ} \times 0.46^{\circ}$ of visual angle) was presented. The locations of the memory test probe were selected randomly from eight possible locations, with the restriction that its location matched that of one of the four memory stimuli on half of the trials. The participants were asked to report whether the empty square was present at the same location as one of the four squares in the memory array.

Visual search task. The search array for each trial consisted of 4,8 , or 12 black items (search stimuli, each $0.46^{\circ} \times 0.46^{\circ}$ of visual angle). Each of the search stimuli was an L-shaped figure, oriented $0^{\circ}, 90^{\circ}, 180^{\circ}$, or $270^{\circ}$ from the upright position. To maintain the same display density, four items were presented in one quadrant, and set size was manipulated by varying the number of quadrants containing four items. The target was an upright L-shaped figure and was presented on half of the trials. The participants were required to make a response to indicate whether or not the target was present as quickly and accurately as possible.

\section{Procedure}

The dual-task, the memory-alone, and the search-alone conditions were tested in separate blocks. Each condition consisted of 10 practice trials and 144 experimental trials. The order of blocks was randomized across participants.

Each participant was required to articulate a sequence of four Korean letters through the entire trial in order to minimize verbal encoding of the memory array (Besner, Davies, \& Daniels, 1981).

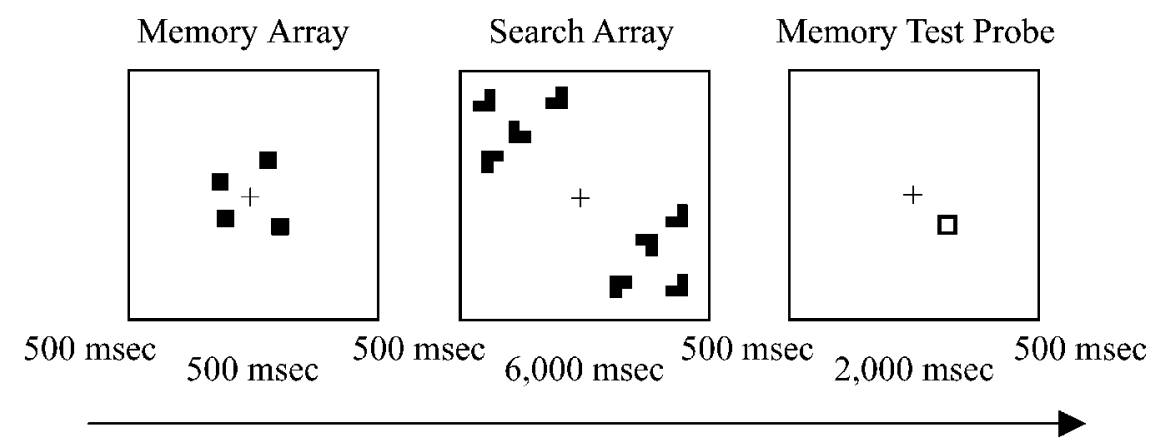

Figure 1. Example of the procedure in the dual-task condition in Experiment 1. The participants were instructed to memorize the locations of four squares in the memory array. The participants reported whether an upright $L$-shaped figure was present in the search array and then reported whether an outlined square in the memory test display was in the same location as one of the four squares in the memory array. 

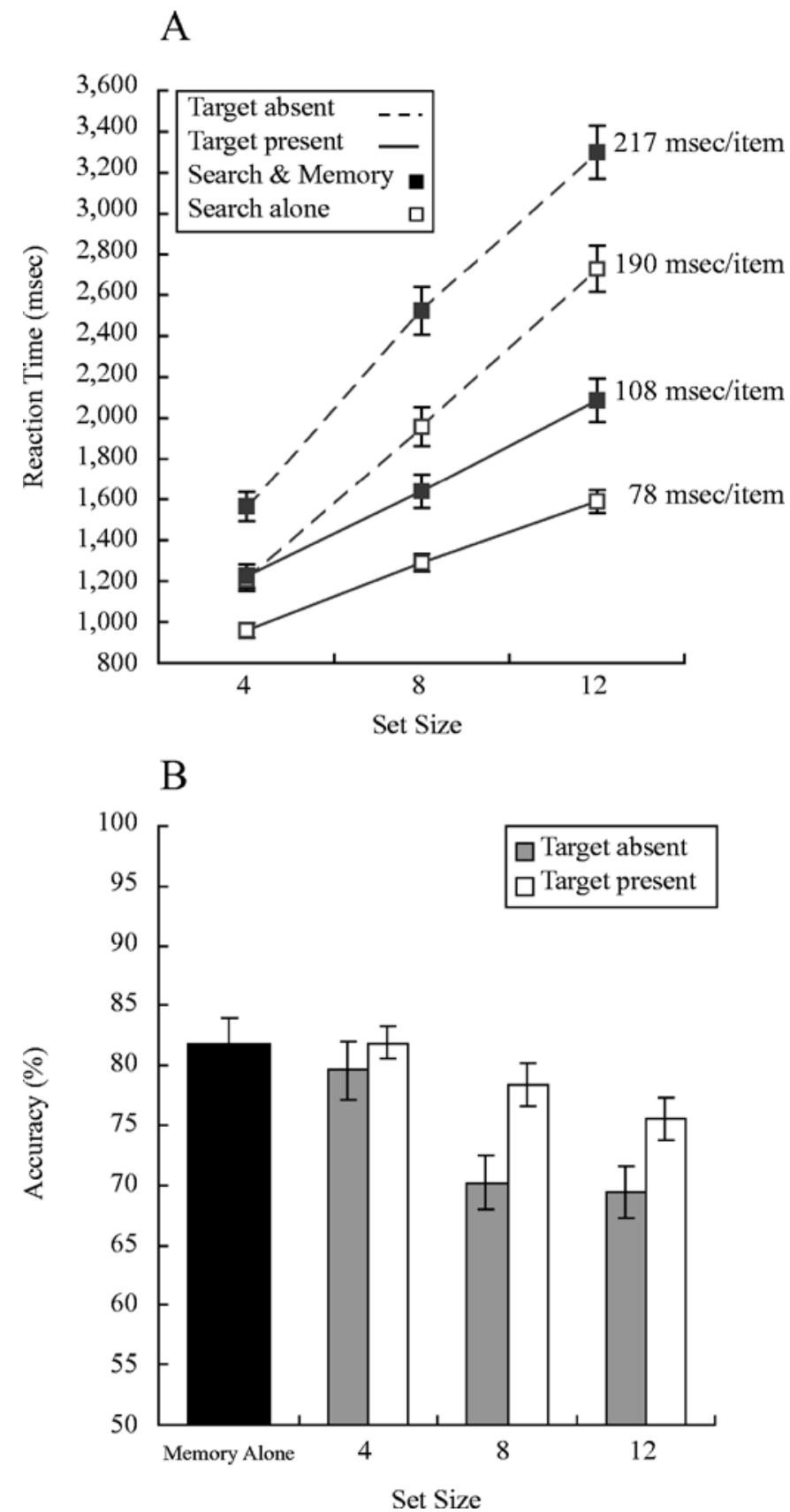

Figure 2. Results in Experiment 1. (A) Mean search reaction times in the dual-task condition and in the search-alone condition. (B) Mean memory accuracy in the dual-task condition and in the memory-alone condition. The error bars represent standard errors.

After the articulation began, the participant initiated each trial by pressing the space bar. In the dual-task condition, a trial began with a 500-msec blank period, which was then followed by the memory array for $500 \mathrm{msec}$. After another 500-msec blank period, the search array was presented for 6,000 msec. After another 500-msec blank period, the memory test array was presented for 2,000 msec. In the memory-alone condition, the search array was replaced by a 6,000 -msec blank period. In the search-alone condition, the mem- ory array and the memory test array were replaced by a 500 -msec blank period or a 2,000-msec blank period, respectively.

\section{Results and Discussion}

\section{Visual Search Performance}

Mean correct search RT and mean search accuracy were analyzed in an analysis of variance (ANOVA) with 


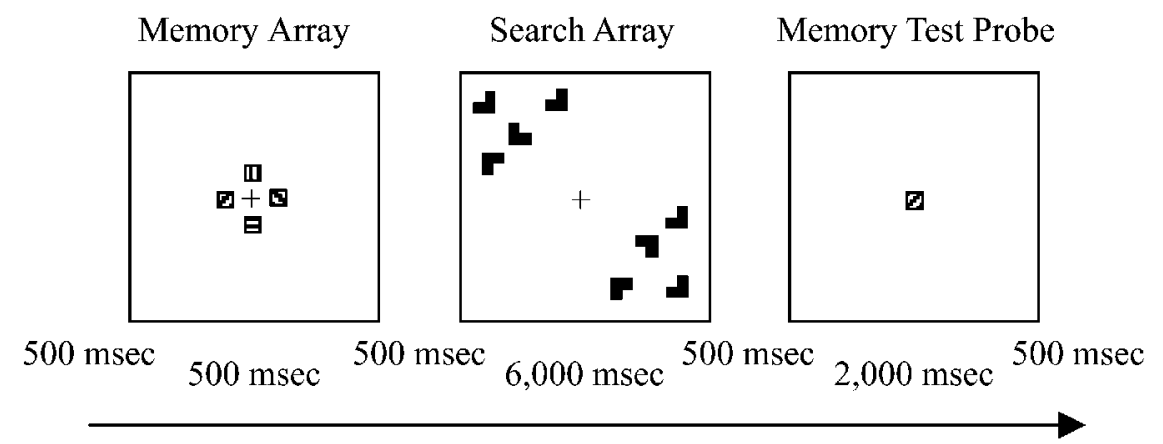

\begin{abstract}
Figure 3. Example of the procedure in the dual-task condition in Experiment 2. The participants were instructed to memorize the colors of four squares in the memory array (each pattern of the squares in this figure represents a different color). The participants reported whether an upright $L$-shaped figure was present in the search array and then reported whether the color of the memory test probe was the same as one of the four colors in the memory array.
\end{abstract}

task (dual-task or search-alone task), set size $(4,8$, or 12), and target (present or absent) as factors.

The RTs for the search tasks, plotted as a function of set size, are shown in Figure 2A. RT increased as set-size increased in all conditions $[F(2,32)=495.48, p<.001]$. Also, RT was greater in the dual-task condition than in the search-alone condition $[F(1,16)=26.63, p<.001]$ and was greater in the target-absent condition than in the target-present condition $[F(1,16)=232.74, p<.001]$. More interesting, the search rate was greater in the dualtask condition $(217 \mathrm{msec} / \mathrm{item}$ in the target-absent condition; $108 \mathrm{msec} /$ item in the target-present condition) than in the search-alone condition $(190 \mathrm{msec} / \mathrm{item}$ in the target-absent condition; $78.6 \mathrm{msec} /$ item in the targetpresent condition), which was confirmed by the significant interaction between task and set size $[F(2,32)=$ $10.37, p<.001]$. The increase of search rate in the dualtask condition indicates that the spatial working memory load impaired the search process. The interactions between target and set size $[F(2,32)=204.44, p<.001]$ and task and target $[F(1,16)=5.37, p<.05]$ also reached significance. The interaction for task, set size, and target was not significant $[F(2,32)=1.16, p>.30]$.

Search accuracy was above $95 \%$ correct for both the dual-task condition and the search-alone condition. The search accuracy difference between the dual-task condition and the search-alone condition was not statistically significant $[F(1,16)=2.92, p>.10]$. An ANOVA of search accuracy revealed a significant main effect of target $[F(1,16)=38.08, p<.001]$ and set size $[F(2,32)=$ $4.67, p<.05]$. The interactions between the factors were not significant (all $p \mathrm{~s}>.15$ ).

\section{Visual working memory performance}

Mean accuracy for the memory tasks, plotted as a function of set size, is shown in Figure 2B. Mean accuracy was lower in the dual-task condition $(76 \%)$ than in the memory-alone condition (82\%). A $t$ test of accuracy in the dual-task condition and the memory-alone condition was significant $[t(16)=2.29, p<.05]$. Moreover, in the dual-task condition, accuracy on memory performance decreased as set size increased. This observation was confirmed by an ANOVA showing a significant main effect of set size $[F(2,32)=15.18, p<.001]$. This set size effect on memory accuracy in the dual-task condition indicates that the search process affected maintenance of spatial information in working memory. The main effect of target was significant $[F(1,16)=18.84$, $p<.001]$, and the interaction between target and set size was not significant $[F(2,32)=0.49, p>.60]$.

\section{EXPERIMENT 2 \\ Nonspatial Working Memory and Visual Search \\ Method}

Whereas in Experiment 1 we investigated spatial working memory, Experiment 2 utilized a nonspatial working memory task. Besides the type of working memory stimuli, all other aspects of Experiment 2 were the same as those in Experiment 1 (see Figure 3).

\section{Participants}

A new group of 17 undergraduate students whose vision was normal or corrected to normal participated for course credit after giving informed consent.

\section{Stimuli and Tasks}

Working memory task. The memory stimuli for each trial were four colored squares. The colors of the filled squares were selected randomly from eight possible discriminable colors (red, yellow, green, blue, violet, brown, black, and white). Each square was located on the top, bottom, left, or right side of fixation, which were $0.68^{\circ}$ of visual angle from fixation. The participants were asked to remember the colors of the four squares in the memory array until the end of the trial.

At the end of each trial, one colored square (memory test probe, $0.46^{\circ} \times 0.46^{\circ}$ of visual angle) was presented at the center of the display. The colors of the memory test probe were selected randomly from eight possible colors, with the restriction that its color should match one of the four memory stimuli on half of the trials. The par- 

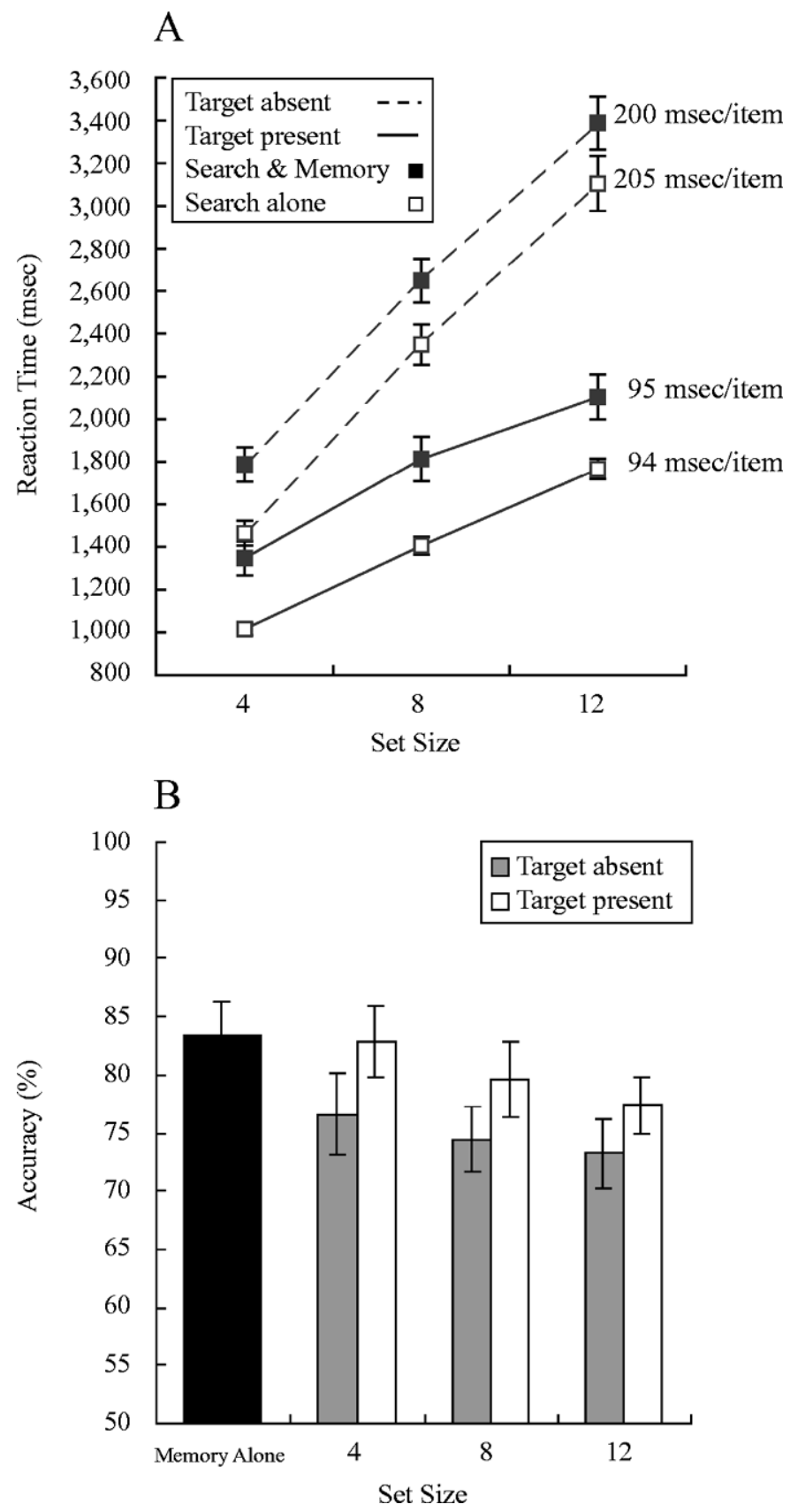

Figure 4. Results in Experiment 2. (A) Mean search reaction times in the dual-task condition and in the search-alone condition. (B) Mean memory accuracy in the dual-task condition and in the memory-alone condition. The error bars represent standard errors.

ticipants were asked to report whether the memory test probe was the same color as one of the four squares in the memory array.

\section{Results and Discussion}

\section{Visual Search Performance}

The search RT and accuracy data in Experiment 2 were analyzed in the same way as those in Experiment 1.
As is shown in Figure $4 \mathrm{~A}$, the main effects of task $[F(1,16)=19.20, p<.001]$, set size $[F(2,32)=527.71$, $p<.001]$ and target $[F(1,16)=250.42, p<.001]$ were significant. However, the search rate was not greater in the dual-task condition $(200 \mathrm{msec} /$ item in the targetabsent condition; $94.6 \mathrm{msec} /$ item in the target-present condition) than in the search-alone condition $(205 \mathrm{msec} /$ 
item in the target-absent condition; $93.8 \mathrm{msec} / \mathrm{item}$ in the target-present condition), showing no significant interaction task and set size $[F(2,32)=0.50, p>.60]$. The interaction of target and set size $[F(2,32)=184.99, p<$ $.001]$ was significant. The interaction between task and target did not approach significance $[F(1,16)=0.93, p>$ .30], and the interaction of task, set size, and target was not significant $[F(2,32)=0.65, p>.50]$.

Search accuracy was above $95 \%$ correct for each set size in the dual-task condition and the search-alone condition. The main effect of task was not significant $[F(1,16)=$ $0.19, p>0.60]$. An ANOVA of the search accuracies revealed significant main effects of target $[F(1,16)=15.31$, $p<.005]$ and set size $[F(2,32)=6.59, p<.005]$. The interactions between the factors were not significant (all $p \mathrm{~s}>0.05)$.

\section{Visual Working Memory Performance}

The memory accuracy data in Experiment 2 were analyzed in the same way as those in Experiment 1. Mean accuracy for the memory tasks, plotted as a function of set size, are shown in Figure 4B. Mean accuracy was lower in the dual-task condition (77\%) than in the memoryalone condition (83\%). A $t$ test of accuracy in the dualtask condition and the memory-alone condition was significant $[t(16)=2.36, p<.05]$. However, the main effect of set size did not approach significance $[F(2,32)=1.63$, $p>.20$, indicating that the search process did not systematically interfere with maintenance of nonspatial information in working memory. The main effect of target was significant $[F(1,16)=6.96, p<.05]$, and the interaction between target and set size was not significant $[F(2,32)=0.14, p>.80]$.

\section{GENERAL DISCUSSION}

In the present study, we investigated the relation between attention and working memory in a combined visual search and working memory task. We found interference between the visual search process and spatial working memory storage: The spatial working memory load impaired search efficiency, and the search process impaired spatial working memory accuracy. These results imply that the visual search process and spatial working memory storage require the same limited-capacity mechanisms.

Interference between visual search and spatial working memory in the present study could result from the common demand for spatial attention. Awh and his colleagues (Awh, Jonides, \& Reuter-Lorenz, 1998) have proposed that spatial attention serves as a rehearsal mechanism for spatial working memory. In their research, choice RTs were shorter when the stimuli were presented at a location held in working memory, and spatial working memory accuracy was impaired when a shift of attention was required by an intervening task. In the same manner, holding of spatial locations in Experiment 1 of the present study was likely to interfere with a visual search process that required shifting spatial attention.
In addition, there is a possibility that spatial information for searched items was transferred into working memory for efficient visual search, so that it interfered with spatial information for the working memory task. Klein (1988) has proposed that memory for previously searched locations inhibits reinspection of objects during serial visual search, and he named this inhibitory effect inhibitory tagging. With increasing search set size, the locations to be remembered for efficient search increase, making the interference between spatial working memory and visual search processes likely to increase.

In contrast to the spatial working memory task, the nonspatial working memory task did not interfere with the visual search process. Taken together, these results suggest that spatial information of searched items is more crucial than nonspatial information when searching a predefined target object efficiently. This is more strongly supported by the cross-experiment analysis that revealed a significant interaction of experiment, task, and set size $[F(2,64)=6.51, p<.005]$. In other words, spatial and nonspatial working memory loads were different in their effects on search efficiency.

The implication of the present study is that, in regard to its interaction with attentional processes, spatial working memory can be distinct from nonspatial working memory. Even though the present study demonstrated a significant interaction of attentional processes only with spatial working memory, it also should be noted that the role of working memory during a visual search task could vary depending on the nature of the visual search task. This would mean that visual information of benefit to a current visual search is likely to be encoded in working memory. For future research, it will be necessary to address the question of which specific properties of various attention tasks require interactions with spatial working memory, nonspatial working memory, or both.

\section{REFERENCES}

Awh, E., Jonides, J., \& Reuter-Lorenz, P. A. (1998). Rehearsal in spatial working memory. Journal of Experimental Psychology: Human Perception \& Performance, 24, 780-790.

BAddeley, A. D., \& Logie, R. H. (1999). Working memory: The multiple component model. In P. Shah \& A. Miyake (Eds.), Models of working memory (pp. 28-61). Cambridge: Cambridge University Press.

Besner, D., Davies, J., \& DAniels, S. (1981). Reading for meaning: The effects of concurrent articulation. Quarterly Journal of Experimental Psychology, 33A, 415-437.

CAVE, K. R., \& Wolfe, J. M. (1990). Modeling the role of parallel processing in visual search. Cognitive Psychology, 22, 225-271.

Chelazzi, L., Duncan, J., Miller, E. K., \& Desimone, R. (1998). Responses of neurons in inferior temporal cortex during memoryguided visual search. Journal of Neurophysiology, 80, 2918-2940.

Chelazzi, L., Miller, E. K., Duncan, J., \& Desimone, R. (1993). A neural basis for visual search in inferior temporal cortex. Nature, 363, 345-347.

Courtney, S. M., Petit, L., Maisog, J. M., \& Ungerleider, L. G. (1998). An area specialized for spatial working memory in human frontal cortex. Science, 279, 1347-1351.

Courtney, S. M., Ungerleider, L. G., Keil, K., \& Haxby, J. V. (1996). Object and spatial working memory activate separate neural systems in human cortex. Cerebral Cortex, 6, 39-49.

DESIMONE, R. (1996). Neural mechanisms for visual memory and their 
role in attention. Proceedings of the National Academy of Sciences, 93, 13494-13499.

Desimone, R. (1998). Visual attention mediated by biased competition in extrastriate visual cortex. Philosophical Transactions of the Royal Society of London: Series B, 353, 1245-1255.

Desimone, R., \& Duncan, J. (1995). Neural mechanisms of selective visual attention. Annual Review of Neuroscience, 18, 193-222.

DownING, P. E. (2000). Interactions between visual working memory and selective attention. Psychological Science, 11, 467-473.

KIM, M.-S., \& CAVE, K. R. (1995). Spatial attention in visual search for features and feature conjunctions. Psychological Science, 6, 376-380.

KIM, M.-S., \& CAVE, K. R. (1999). Top-down, and bottom-up attentional control: On the nature of interference from a salient distractor. Perception \& Psychophysics, 61, 1009-1023.

KiM, M.-S., \& RoberTSON, L. C. (2001). Implicit representations of space after bilateral parietal lobe damage. Journal of Cognitive Neuroscience, 13, 1080-1087.

KLEIN, R. M. (1988). Inhibitory tagging system facilitates visual search. Nature, 334, 430-431.

LUCK, S. J., \& VOGEL, E. K. (1997). The capacity of visual working memory for features and conjunctions. Nature, 390, 279-281.
Mccarthy, G., Puce, A., Constable, R. T., Krystal, J. H., Gore, J. C., \& Goldman-RAKIC, P. S. (1996). Activation of human prefrontal cortex during spatial and object working memory tasks measured by functional MRI. Cerebral Cortex, 6, 600-611.

SHORE, D. I., \& KLEIN, R. M. (2000). On the manifestations of memory in visual search. Spatial Vision, 14, 59-75.

Smith, E. E., Jonides, J., \& KoEPPE, R. A. (1996). Dissociating verbal and spatial working memory using PET. Cerebral Cortex, 6, 11-20.

Smith, E. E., Jonide S, J., Koeppe, R. A., Awh, E., Schumacher, E. H., \& Minoshima, S. (1995). Spatial versus object working memory: PET investigations. Journal of Cognitive Neuroscience, 7, 337-356. TREISMAN, A. M., \& Gelade, G. (1980). A feature integration theory of attention. Cognitive Psychology, 12, 97-136.

Wolfe, J. M. (1994). Guided Search 2.0: A revised model of visual search. Psychonomic Bulletin \& Review, 1, 202-238.

Woodman, G. F., Vogel, E. K., \& LUCK, S. J. (2001). Visual search remains efficient when visual working memory is full. Psychological Science, 12, 219-224.

(Manuscript received December 2, 2002; revision accepted for publication September 26, 2003.) 